\title{
Zukunft modellieren: Science Fiction als soziale Simulation von Hyperobjekten
}

\author{
Lars Schmeink $^{1}$ \\ ${ }^{1}$ HafenCity Universität, Überseeallee 16, 20457 Hamburg; lars.schmeink@hcu-hamburg.de
}

\begin{abstract}
Climate change, pandemics, digitalization - there are many hyperobjects, as Timothy Morton [1] describes them, that are too big and too complex for any one of us to fully grasp. On the one hand, these objects affect all of us, creating a need for "the immanence of thinking to the physical," while at the same time being so large, complex, and beyond our metalanguage capabilities that they create an "absence of anything meaningfully like a 'world."' The language and data of science do not do justice to the personal appropriation of these issues - we are not experiencing $r$-factors and infection vectors; we are personally affected by living with social distancing measures and the breakdown on social contracts.

So, in order to grasp these hyperobjects and make sense of what the future will hold, the paper argues, we need to move beyond the modeling of pure scientific data and instead turn towards the social, political, and cultural aspects of each of these hyperobjects. We need to simulate the impact of scientific facts onto the fabric of our lives. And for this purpose, fictions are needed that leave behind the data driven empiricism and instead model a new social reality - and the paper argues, this is the purview of science fiction.
\end{abstract}

\section{Einleitung}

Die Zukunft zu modellieren ist nicht einfach. Für bestimmte Fragen scheint dies jedoch mit Hilfe von Simulationen und Modellen besser möglich als für andere. So haben Carl Benedikt Frey und Michael Osborne an der Universität Oxford die Entwicklung von Automatisierung und Digitalisierung im Bereich der Arbeitsprozesse untersucht und Berechnungen erstellt, welche Berufe auch in Zukunft mit hoher Wahrscheinlichkeit von Menschen ausgeübt werden. Aber kaum ein Modell beschäftigt sich damit, welche privaten und persönlichen Konsequenzen diese Entwicklung für die tausenden Betroffenen haben wird und was diese Veränderungen dann gesellschaftlich auslösen. Soziologische Studien können hier vornehmlich stattfindende oder abgeschlossene Umbrüche dokumentieren, bieten aber kaum die Möglichkeit zukünftige Veränderungen zu antizipieren. An dieser Stelle ist es also hilfreich sich auf die Kultur als Gradmesser von Hoffnungen und Sorgen einer Gesellschaft zu berufen und mit Hilfe der Science Fiction (SF) - eines kulturellen Modus, der sich dezidiert mit technologischem Wandel und dessen sozialen Folgen beschäftigt mögliche Szenarien der Zukunft zu entwickeln. Für die folgenden Überlegungen nutze ich daher eine für die Natur- und Ingenieurswissenschaften unorthodoxe Auslegung von Modellen, die uns aber dennoch dabei helfen kann, gesellschaftliche Zukunftsbilder begreifbar zu machen. Ich behaupte, die Science Fiction kann uns Modelle für das liefern, was anderweitig nur schwer greifbar ist. ${ }^{1}$

Bevor ich aber zur SF und ihren Spezifika komme, möchte ich kurz erläutern, wo die Verbindung von Fiktionalität und Modellierung liegt. Zwei Punkte sind dabei wichtig. Zum einen, dass Kultur Realität abbildet. Zum anderen, dass Fiktionalität nicht gleichzusetzen ist mit Täuschung, Lüge oder Erfindung, sondern eine Beschreibung möglicher Handlungen, Zusammenhänge, oder Zustände darstellt. Zum ersten Punkt: Kultur ist ein Modell unserer Realität. Sie beschreibt, mal mehr, mal weniger detailliert, was potentiell passieren kann. Dabei nutzt sie mitunter auch Abstraktionen, Verkürzungen, oder Verfremdungen. Dies lässt sich beispielsweise daran festmachen, dass wir literarische Verkürzungen mit uns bekannten Mustern auffüllen. Wenn also in einem Roman der Satz steht „Wir gingen zum Abendessen in unser Lieblingsrestaurant“, dann können wir mit einiger Sicherheit trotz des niedrigen Detailgrades und der starken Verkürzung relativ genau sagen, wie der Abend abgelaufen ist zumindest auf der rein technischen Seite der Handlungen. Im Großen und Ganzen dürfte etwa folgendes passiert sein: Ankunft im Restaurant, Platz nehmen am Tisch,

\footnotetext{
${ }^{1}$ Teile dieses Beitrags sind in leicht abgewandelter Form bereits erschienen. [2]
} 
Auswahl, Anlieferung und Verzehr der Speisen, Bezahlung der Dienstleistung, Verlassen des Restaurants. Dazwischen gibt es viele Faktoren, die wir nicht genau bestimmen können. Und es gibt mögliche Abweichungen von den wahrscheinlichen Handlungen. Aber weil im Roman keine weiteren Details stehen, gehen wir beim Lesen davon aus, dass Abweichungen und Details nicht relevant für unsere weitere Auseinandersetzung mit dem Abend sind. Wir haben ein Modell des Restaurantbesuchs, abstrakt und detailarm, aber ein Modell.

Ähnlich funktioniert diese Realitätsmodellierung von Kultur in Filmen oder Serien. Wir sehen beispielsweise die Figuren in ein Auto steigen und die Straße entlang aus dem Bild fahren. In einer späteren Szene sehen wir dann dasselbe Auto anhalten und die Figuren steigen wieder aus. Auch hier ist die Abstraktion des Modells recht hoch, die Detaildichte ist gering. Aber doch gehen wir davon aus, dass zwischen beiden Szenen eine Fahrt liegen muss, dass das Auto an Ampeln gehalten hat, beschleunigt und gebremst hat, abgebogen ist, Spuren gewechselt hat - dass also all das passiert ist, was auf dem Weg von A nach B mit einem Auto passiert. Wiederum steht uns ein Modell abstraktes und detailarmes Modell einer Autofahrt zur Verfügung, was uns in wenigen Bildern vermittelt wurde.

Der zweite Punkt - Fiktionen sind nicht mit Täuschungen gleichzusetzen - ist hier auch schon angerissen. Die beschriebenen Figuren mögen nicht real sein, ihre Handlungen aber sind es: Restaurantbesuche und Autofahrten sind Teil unserer Realität, somit in ihrer Abstraktion keine Täuschungen. Weiter noch: Fiktionen bestimmen unser Miteinander. Wir sehen in Fiktionen Muster und Handlungen, die wir dann als Modell für die Realität nehmen und in dem Moment kopieren, da wir keine eigene Erfahrung mit der jeweiligen Handlung haben. Ein Beispiel für dieses Phänomen ist, dass viele Menschen vor deutschen Gerichten auf den „Einspruch“ warten, den es hierzulande in dieser Form nicht gibt, der aber auf USamerikanischen Fiktionen in unserem Modell einer Gerichtsverhandlung vorkommt. Fiktion bestimmt also zu einem gewissen Grad unsere Erwartung an die Realität.

Die Funktion von Fiktionen unsere wahrgenommene Wirklichkeit möglichst nah abzubilden nennt man in der Literaturwissenschaft Mimesis. Mimetische Darstellungen in Literatur und Film sind das, was man im allgemeinen Sprachgebrauch auch als, realistisch“ ansieht. Das ist oftmals der Fall, wenn etwa Zwischenmenschliches modelliert werden soll - also wie Menschen sich lieben, sich begegnen, aneinander verzweifeln. Oder bei historischen
Ereignissen, die im Nachgang möglichst getreu der Realität nacherzählt werden sollen. Aber was passiert, wenn wir komplexe und ,ungreifbare‘ Dinge wie den Klimawandel, die Digitalisierung, oder die Globalisierung abzubilden versuchen? Mein Argument ist, dass hierzu die Science Fiction nötig ist. Kultur kann auch diese komplexen Objekte sinnvoll modellieren und so einem Massenpublikum erzählend näherbringen, wenn sie dafür auf die SF als Modus zurückgreift.

Die Literaturwissenschaftlerin Seo-Young Chu [3] argumentiert, dass die Science Fiction besonders leistungsstark darin sei, Welt zu modellieren. Aber SF sei nicht etwa der Gegenpol zur mimetischen Hochliteratur, sondern vielmehr müsse man $\mathrm{SF}$ als eine mimetische Form verstehen, die Abbilder bestimmter besonders schwer zu greifender Wirklichkeitsbezüge, sogenannter „Cognitively estranging referents“ (5), generieren will. Also Bezüge zu Objekten, die für den Menschen nicht vollständig kognitiv erfahrbar sind und nur mit einem gewissen Maß an zusätzlicher kreativer Energie beschrieben werden können. Chu situiert diese im Mittel eines Spektrums zwischen Wissbarem und Unwissbarem (6). Auf der Seite des Wissbaren finden sich Alltagsgegenstände wie Werkzeuge, Essen, Kleidung usw., die eine realistische Literatur mit hoher Präzision mimetisch darzustellen vermag. Auf der Seite des Unwissbaren finden sich Konzepte, die außerhalb der menschlichen Kognition liegen und mimetisch überhaupt nicht darstellbar sind, wie etwa die Zeit des Urknalls oder Erfahrungen jenseits des Todes (7). In der Mitte dieses Spektrums aber finden sich Objekte, die „kognitiv verfremdend“ sind erkennbar, allerdings kaum erfahrbar: „the sublime (e.g., outer space), virtual entities (cyberspace), realities imperceptible to the human brain (the fourth dimension), phenomena whose historical contexts have not yet been fully realized (robot rights), and events so overwhelming that they escape immediate experience (shell shock)“ (7). Die Bezugnahme auf diese Objekte in der Wirklichkeit ist nicht vollständig möglich und so bedürfen sie, wenn man so will, einer höheren literarischen Energie für eine mimetische Annäherung.

Die SF sorgt also dort für eine Annäherung an mimetische Repräsentation, wo die komplexen Wirklichkeitsbezüge für die Leser*in sonst nicht erfahrbar wären. Ich würde sogar so weit gehen, zu behaupten, dass viele Aspekte unserer aktuellen Lebensrealität solche Komplexität aufweisen und eben einer SF-mimetischen Darstellung bedürfen. Chu führt beispielsweise das Leben in ei- 
ner stark medialisierten Welt an, in der die Grenzen zwischen virtueller und materieller Existenz dank allgegenwärtiger, digitaler Technologien verschwimmen (9). Und die schwer durchdringlichen ökonomisch-politischen Realitäten der spätkapitalistischen Weltwirtschaft mit ihren „too big to fail“-Banken, digitalen Monopolisten und globalisierten Märkten scheinen ebenfalls eine ideale Ausdrucksform in der SF $\mathrm{zu}$ finden. Im Folgenden möchte ich daher einige Beispiele solcher SF-nahen Objekte diskutieren. Im heutigen politischen Alltag lässt sich gut beobachten, wie die Komplexität einer globalisierten Welt selbst für Experten undurchschaubar geworden ist. Gerade in der aktuellen Corona-Situation zeigt das Fehlen von Auffangmechanismen und Krisenplänen, wie wenig tiefgehendes Verständnis von Marktzusammenhängen in Wirtschaft und Politik vorhanden ist. Die Komplexitäten des globalen Kapitalismus haben sich durch die Krisensituation als unwägbar erwiesen, besonders weil plötzliche Rückfälle in Zeiten strikter Grenzkontrollen und eingeschränkter Freizügigkeit in keinem Zukunftsszenario verlässlich berechnet worden sind. Und auch gesellschaftlich gesehen sind die Auswirkungen der Globalisierung komplex und überall, wenn auch diffus, spürbar - Automatisierung und Digitalisierung lassen auch in der bislang verschonten westlichen Welt die bestehenden Sicherheiten zusammenbrechen. Die Pandemie hat diesen Entwicklungen nur weiter Vorschub geleistet. Migrationsbewegungen und ökologische Veränderungen werden in Zukunft zu den wirtschaftlichen Prozessen hinzuaddiert werden müssen und lassen die Globalisierung so dem werden, was Timothy Morton [1] als Hyperobjekt bezeichnet.

Morton sieht Hyperobjekte als Objekte, die im Maßstab menschlicher Erfahrungen zu groß und zu weitreichend sind, um vollständig erfasst werden zu können (1). Er zählt beispielsweise das Sonnensystem, die Menge allen nuklearen Materials auf der Erde, die Lebensdauer von Styropor oder die globale Erderwärmung dazu. Ich möchte dazu auch die Globalisierung ergänzen, die Prozesse der Digitalisierung und deren Einfluss auf die Arbeitsmärkte, den Klimawandel, oder eben eine Pandemie wie Corona. Hyperobjekte sind, laut Morton, „viscous“ (also klebrig oder zähflüssig) und man kann ihnen nicht aus dem Weg gehen. Hyperobjekte lassen sich nicht ignorieren: „Every attempt to pull myself free by some act of cognition renders me more hopelessly stuck to hyperobjects“ (29). Wir müssen uns mit diesen komplexen und allgegenwärtigen Themen beschäftigen. Aber sie lassen sich nicht gut beschreiben, nicht in klar greifbare Kategorien packen. Naturwissenschaftliche Modelle können einzelne Aspekte solcher Hyperobjekte visualisieren, aber man braucht spezielles Wissen, um die Fülle an Daten in Zusammenhang zu bringen und korrekt zu interpretieren. Die Wissenschaftskommunikation ist täglich damit beschäftigt, Narrative zu präsentieren, Beispiele zu liefern und für die Öffentlichkeit neue Ankerpunkte zu schaffen, um Befunde und Erkenntnisse über Hyperobjekte zu vermitteln. Doch wie sehr solche datenbasierte Vermittlung scheitern kann, lässt sich aktuell an der weltweit wachsenden Zahl der Corona-Leugner erkennen. Die Science Fiction macht jedoch mit Hilfe von Literatur, Film und Fernsehen Hyperobjekte für die Allgemeinheit erfahrbar, wie ich nun an einigen Beispielen aufzeigen möchte.

\section{Globalisierung: William Gibsons The Peripheral und Agency}

In seinen aktuellen Romanen The Peripheral [4] und Agency [5] beschreibt William Gibson das Hyperobjekt der Globalisierung, indem er dessen hyperbolisch erscheinende Komplexität literarisch vereinfacht und die Welt buchstäblich zu einem Spielball der Mächtigen macht. The Peripheral beschreibt zwei Zukunftsperspektiven: die eine in der nahen Zukunft, in einer ländlichen Gegend in den USA. Hier arbeitet die Protagonistin Flynne in einer von der Digitalisierung geprägten Welt für eine kolumbianische Firma als Sicherheitskraft. Doch statt in physischer Präsenz muss Flynne ihr Schutzobjekt per virtueller Realität überwachen. Ihre Arbeit gleicht einem Computerspiel, und als in dieser virtuellen Welt ein Mord geschieht, den Flynne beobachtet, gerät ihr Leben ganz real aus den Fugen.

In der anderen Zukunftsperspektive, mehr als 70 Jahre später, hat sich das weltweite System der Märkte komplett verändert. Ein globaler Zusammenbruch (sowohl ökologisch als auch ökonomisch) hat die Menschheit dezimiert; Fragmente der bestehenden Welt konnten mit Hilfe von Nanotechnologie neu aufgebaut werden, aber der Verlust der Erde als natürliche Ressource ist deutlich spürbar. In einem künstlich wiedererschaffenen London des 22. Jahrhunderts lebt eine reiche Elite, die vor allem mit Machtspielen und politischer Einflussnahme beschäftigt ist. Arbeitskraft, die für den Erhalt ihres Lebensstandards notwendig ist, ist entweder automatisiert oder wird aus der Vergangenheit eingekauft und 
virtuell erbracht. Mittels Quantencomputern ist es technologisch möglich, sogenannte Kontinua zu erschaffen, Abzweigungen möglicher Welten, in denen die Zukunft die Vergangenheit beeinflusst und nach Belieben manipuliert. Flynne ist Teil einer solchen alternativen Zeitlinie, die von den reichen Eliten als Spielball für ihre Intrigen genutzt wird. Der Mord, den sie in der virtuellen Realität gesehen hat, ist im London des 22. Jahrhunderts real geschehen.

Auch in Agency geht es um diese alternativ-geschichtlichen Welten. Wiederum wird ein solcher Stub (also Stummel) - wie die Zukunft des 22. Jahrhunderts aus privilegierter Sicht die abweichenden Zeitlinien nennt - von zukünftigen Eliten als Experimentierfeld genutzt. Die Welt des Stubs steht kurz vor dem Atomkrieg, den Präsidentin Hillary Clinton mit allen Mitteln zu verhindern versucht. Der Stub gilt als wichtig, weil er die am weitesten zurückreichende Abweichung von der Zeitlinie darstellt. In ihm könnte sich zeigen, ob es möglich gewesen wäre, dass der ökonomische und ökologische Kollaps der Welt hätte verhindert werden können. Deswegen nutzen die Mächtigen der Zukunft eine militärisch entwickelte KI, um die Machtverhältnisse der Vergangenheit zu stabilisieren und den Krieg zu verhindern. Dabei wird aber vor allem deutlich, dass die Manipulation der Vergangenheit eine nostalgische Beschäftigung ist: also der Versuch, die in der eigenen Zeitlinie katastrophal zerstörten Systeme in anderen Zeitlinien zu erhalten und den Kollaps zu verhindern. Ein bestimmter Teil der Zukunft trauert dem heutigen Machtsystem, den freien Märkten und dem technologischen Erfindergeist hinterher - aus Sicht Gibsons haben wir heute das Potential, noch alles zum Besseren zu kehren.

Die Kontinua repräsentieren mimetisch das Hyperobjekt der Globalisierung in den Romanen. Die Mächtigen des 22. Jahrhunderts vermögen aufgrund ihres technologischen Fortschritts in der Zeit zurückzureichen und die Menschen dort zu instrumentalisieren. Dass in den Romanen die Nutzung von Kontinua als dekadente Subkultur von Oligarchen und Aristokraten aufgezeigt wird, die alternative Zeitlinien zur Unterhaltung oder Ausbeutung generieren, lässt tief blicken. Die Lebensbedingungen in den Stubs sind nicht von Bedeutung; die Menschen sind Figuren in einer virtuell erlebten ökonomischen Simulation. Das Leben in einem Stub wird von allwissenden und zu allem fähigen Mächten kontrolliert - ein Verständnis der eigenen Situation bleibt den Menschen verwehrt. Der Stub hat in etwa den Stellenwert eines komplexen wirtschaftlichen, gesellschaftlichen Computerspiels. Seine
Welt hat keinen Nutzen in direktem Bezug auf die ihn generierende Realität des 22. Jahrhunderts - Konsequenzen eingreifender Handlungen verbleiben im Stub und erreichen nicht deren Erschaffer.

Bezeichnend ist hierbei, dass die Machtausübung auf die Vergangenheit, wie auch der Austausch von Informationen zurück in die Zukunft sich einzig auf digitale Transaktionen beschränkt. Angesichts der fortgeschrittenen Digitalisierung, sowohl in Hinsicht auf finanzielle aber auch auf materielle Prozesse, ist dies aber kein Hindernis für direkte Einflussnahme. Politische Gefälligkeiten lassen sich in der Vergangenheit ebenso digital erkaufen wie wirtschaftliche Macht (etwa durch Technologiepatente) oder materielle Objekte - neue Waffen können z.B. dank 3D-Druckern durch die Zeit gelangen.

Der von Gibson in den Romanen beschriebene Gegenstand ist nicht die lokale Ausprägung von Globalisierung, wie sie eine realistische Literatur darzustellen vermag. Statt ihre Auswirkungen in den Schicksalen einer durchschnittlichen, amerikanischen Familie aufzuzeigen, widmet sich Gibson der für den Einzelnen verborgenen Struktur der Globalisierung. Tatsächlich verweist Morton [1] darauf, dass Hyperobjekte „non-local“ sind und nicht mittels ihrer „local manifestations“ (1) begriffen werden können. Es bedarf also der Konstruktion von Kontinua als Miniaturwelten, um die systemische Ausbeutung ,verschwendeter Leben', wie der Soziologe Zygmunt Bauman [6] sie nennt, in der Globalisierung darzustellen. Was Bauman in seiner Studie auf Länder des globalen Südens bezieht und als „,unintended and unplanned 'collateral casualties' of economic progress“ (39) bezeichnet, trifft auch heute schon auf Teile der Bevölkerung der USA oder Europas zu, die als Kollateralschäden einer Globalwirtschaft von einer gesellschaftlichen Beteiligung ausgeschlossen sind. Das SF-Erzählen Gibsons ermöglicht hier also das abgeschieden ländlich verarmte Leben (etwa in West Virginia mit seinen geschlossenen Kohleminen, oder im Rust Belt, wo die Stahlwerke nicht mehr konkurrenzfähig sind) mit seinen ökonomischen Realitäten in die Machtstrukturen einer globalen Wirtschaft einzuordnen. Es erlaubt ein Modell zu erstellen, dass das menschenverachtende Profitstreben heutigen Handelns aufzeigt. Und es verdeutlicht dessen transformativen Einfluss, sei es durch globale Migrationsbewegungen oder in dem sozialen Aufbegehren abgehängter Teile westlicher Gesellschaften. 


\section{Klimawandel und soziale Ungleich- heit: Neill Blomkamps Elysium}

Natürlich muss nicht immer ein einzelnes Hyperobjekt so zentral von einem SF-Text modelliert werden. In einigen Fällen bestimmen die Hyperobjekte den Hintergrund vor dem ein Text spielt, in anderen sind verschiedene Objekte abgebildet und verbinden sich zu einem komplexen Gefecht. So verhandelt der Film Elysium von Neill Blomkamp [7] diverse Hyperobjekte und deren gemeinsame Wirkung auf die Menschen. Der Film spielt im Jahr 2154 und zeigt schon in seiner Anfangssequenz die Folgen heutiger gesellschaftlicher Herausforderungen.

Die den Film eröffnende Kamerafahrt führt über ein Flussgebiet, das mit Müll verseucht ist und wie eine Industriebrache wirkt: eine unwirtliche Landschaft, die thematisch bereits in den ersten Sekunden auf Umweltverschmutzungen hinweist. Es folgt ein Überflug über ein Stadtgebiet mit dicht gedrängten Gebäuden, die in keinem guten Zustand sind und eher ärmlich wirken. Die Einblendung eines Zwischentitels bestätigt die Themen, die hier verhandelt werden: die Welt ist verseucht, verschmutzt, und leidet an der Überbevölkerung. Im Hintergrund des Stadtbildes steigen schwarze Rauchsäulen auf. Das typisch aufgeräumte Grid amerikanischer Städte ist einem urbanen Chaos zusammengewürfelter Häuser gewichen. Es sind Bilder, die westliche Zuschauer*innen aus den Slums des globalen Südens kennen. Mit einem Schnitt wechselt die Szene zu Hochhäusern, zerfallen von der Zeit, zerfressen von den Umweltgiften, überwuchert, rauchend. Man erkennt die Skyline von Los Angeles mit der runden Form des US Bank Towers in der Mitte und daneben den markanten Stufen des Citibank Towers. Danach folgt ein Schnitt zur Erdansicht aus dem All und ein weiterer Zwischentitel mit der Einblendung, dass die reichsten Bürger der Erde sich vom Planeten abgewendet und ihre Art zu leben auf einer Raumstation erhalten haben. Die Raumstation Elysium ist der Rückzugsort all derer, die sich die Bürgerschaft dort leisten können. Die Kamerafahrt durch die Ringstation zeigt grüne Gärten, klare Seen und große Villen, die reichlich Platz bieten. Neben der Umweltverschmutzung steht also vor allem auch die Ungleichheit des sozialen Miteinanders im Mittelpunkt des Films. Schon in dieser Eröffnungssequenz sehen wir die Extrapolation dessen, was heute dank segregierter Bebauung und der Institutionalisierung von Gated Communities Realität ist. Die Folgen einer durch Industrialisierung ausgelösten Umweltzerstörung und des Klimawandels tragen nicht alle Menschen gleichermaßen, sondern vor allem die Nicht-Privilegierten. Reichtum und Macht helfen dabei, diesen Konsequenzen zu entgehen.

Die Handlung des Films stellt diesen Konflikt zwischen den machtlosen Massen auf der Erde und den wenigen Mächtigen in Elysium in den Mittelpunkt, konzentriert sich aber nicht auf politische Aussagen zur Demografie oder die ungleiche Wohlstandsverteilung. Viel mehr zeigt der Film eine actionlastige Handlung um den Tagelöhner Max (Matt Damon), der nach einem Arbeitsunfall einen Weg nach Elysium sucht, um sich dort dank besserer medizinischer Versorgung - von einer tödlichen Strahlendosis heilen zu lassen. Dabei sind die sozial-kritischen Aussagen des Films deutlich spürbar und modellieren ein Bild der Zukunft, das sich klar benennen lässt. So sind schon das Setting und seine Darstellung aufschlussreich. Die den Film eröffnenden Bilder von Los Angeles stehen in Kontrast zu dem Glamour, mit dem das Publikum die Pazifikmetropole sonst im Rahmen von Filmen verbindet. Die Darstellung der Stadt wirkt farblich verwaschen, staubig, in Erdtönen gehalten und in klarem Widerspruch zu den kontrastreichen Blauund Grüntönen Elysiums. Das Los Angeles des Jahres 2154 leidet unter Dürre, Verschmutzung, und massiver Hitze - was Filmemacher Blomkamp bildlich umsetzt, in dem die Szene in Mexiko Stadt gedreht hat. In dieser filmisch-geografischen Verschiebung liegt die Erkenntnis, dass der Klimawandel vor dem Reichtum des heutigen L.A. keinen Halt machen wird und die MojaveWüste weiter nach Westen vorrücken wird.

Mit dem Klimawandel verbunden ist die Überbevölkerung, die sich im Film auch konkret in der Frage um Arbeit und Lebensunterhalt zeigt. So ist Max ein Tagelöhner, der einfache, ungelernte Tätigkeiten in einer Fabrik verrichtet und sich damit schon zu den wenigen Glücklichen schätzen kann, die überhaupt einen Job haben. Die meisten Tätigkeiten sind automatisiert worden und Menschen sind nur dort noch tätig, wo sie billiger einzusetzen sind als Maschinen. Der Soziologe Peter Frase [8] argumentiert: „the existence of a large pool of unemployed and low-wage workers operates as a disincentive for employers to automate. After all, why replace a worker with a robot, if the worker is cheaper?“ (13). In der Welt von Elysium bedeutet dies, dass der Überhang an menschlicher Arbeitskraft nichts wert ist und Arbeiter*innen jegliche Rechte verloren haben. Erscheint Max nicht zur Arbeit oder tut nicht das, was man von ihm verlangt, gibt es jederzeit tausende Anderer, die seinen Job machen würden. Und so wird Max vom Vorarbeiter 
in die Strahlenkammer geschickt, um eine Fehlfunktion zu richten, dort durch die Automatik eingeschlossen und mit einer tödlichen Dosis bestrahlt. Das Unternehmen sieht keine Verantwortung für ihn und ersetzt seine Arbeitskraft sofort. Er wird mit Hospiz-Medikation zum Sterben nach Hause geschickt. Sein Leben ist nur etwas wert, wenn er dafür arbeiten kann. Dabei gäbe es für die Mächtigen in Elysium sehr wohl medizinische Versorgung, die Max retten könnte - nur hat er in der stratifizierten Gesellschaft kein Anrecht auf eine solche Behandlung.

Der Film folgt nun Max’ Bestrebungen nach Elysium zu gelangen und sich zu heilen. Dabei wird er zum Revolutionär, dem es gelingt, die Abschottung Elysiums zu durchbrechen und mit einem Sicherheitscode die Algorithmen der automatisierten Versorgung Elysiums neu zu programmieren. Durch eine Änderung im System gelingt es Max, die Rechte auf Sicherheit, Gesundheit, und Grundversorgung auf alle Menschen auf der Erde zu übertragen. Die automatisierten Systeme erkennen die Not der Erdbewohner und senden Hilfstransporte aus ein gleichberechtigtes und vor allem grundversorgtes Leben ist möglich. Nicht ein Mangel an Ressourcen ist also der Grund für die soziale Spaltung, vielmehr ist diese politisch von den Mächtigen Elysiums gewollt, um nicht an Status zu verlieren.

Für Frase [8] stellt diese Vision einer, trotz ausreichender Ressourcen für alle, immer noch hochgradig ungleichen Gesellschaft einen möglichen Extrempunkt unserer sozialen, politischen und ökonomischen Zukunftsentwicklung dar. Er sieht darin die konsequente Weiterführung heutigen Machtdenkens: „To the extent that the rich are able to maintain their power, we will live in a world where they enjoy the benefits of automated production, while the rest of us pay the costs of ecological destruction-if we can survive at all“ (29). Mehr noch, in der logischen Konsequenz einer automatisiert betriebenen Welt, benötigen die Reichen die Armen nicht mal mehr für ihre Arbeitskraft oder ihren Konsum - Frase nennt diese Zukunftsvision „Exterminism“ (29), weil sie den Tod des größten Teils der Weltbevölkerung billigend in Kauf nimmt oder gar absichtlich vorantreibt.

In einem Film wie Elysium sehen wir also mögliche Szenarien einer Entwicklung gleich mehrerer Hyperobjekte - das Voranschreiten des Klimawandels, das ein Leben auf dem Planeten einschränkt und soziale Probleme verschärft; die hohe soziale Ungerechtigkeit durch die Verteilung von Geld und Macht an wenige, die damit alle politischen Entscheidungen beeinflussen können und sich so von anderen Problemen freikaufen; und die $\mathrm{Au}-$ tomatisierung von Arbeit, die ohne soziale Ausgleichsmaßnahmen die Lebensgrundlage von Millionen Menschen in Frage stellt. All diese Themen sind für uns nur schwer greifbare Hyperobjekte, aber im Film werden sie in der Darstellung von Welt modelliert und somit am Schicksal der Figuren deutlich erlebbar.

\section{Pandemie: Von 28 Weeks Later bis Code 46}

Aber wenden wir uns doch noch mal dem Thema zu, dass uns alle in den letzten Monaten so stark beschäftigt hat und für dessen Einfluss auf uns als Gesellschaft wir bis heute keine guten Modelle haben. Oder? Ich würde zum Abschluss meiner Überlegungen provokativ behaupten, dass wir alle uns auf Corona und die daraus folgenden sozialen Aufwühlungen hätten vorbereiten können, wenn wir bei Zombie-Fiktionen besser aufgepasst hätten. Dabei geht es mir nicht um den offensichtlichen Unterschied, dass Corona zum Glück keine Toten wieder auferstehen lässt. Dennoch sind in Zombie-Fiktionen soziale Verhaltensmuster aufgegriffen worden, die uns in den Wochen des Lockdowns und während der anschließenden Lockerungen begegnet sind.

Dass Zombies ein ideales Vehikel sind, um über Maßnahmen des Seuchenschutzes aufzuklären, bewiesen die US-amerikanischen Centers for Disease Control and Prevention [9], als sie Infomaterial zur „Zombie Apocalypse“ herstellten und damit auf eine „Preparedness“ der US-Bevölkerung hinarbeiteten. Eine der Überschriften der Website lautet „Never Fear - CDC is Ready“ und versucht herauszustellen, wie wichtig die wissenschaftliche Expertise und die klare Umsetzung der Seuchenbekämpfungspläne durch die CDC ist [10]. Angesichts der heutigen Situation einer massiven Einschränkung und Manipulation der Arbeit der CDC aus politischen oder ökonomischen Gründen durch das Weiße Haus ist dies natürlich höchst ironisch.

Wer aber Filme wie 28 Weeks Later [11] oder Resident Evil: Apocalypse [12] gesehen hat weiß, dass auch eine Pandemie sich machtpolitisch nutzen lässt. In 28 Weeks Later entscheidet die NATO, obwohl noch nicht viel über das in Großbritannien ausgebrochenen Virus bekannt ist und keineswegs alle Infektionsherde unter Kontrolle sind, die britischen Inseln wieder neu zu besiedeln, um das politische Signal zu geben, man habe das Virus im Griff. Und in Resident Evil: Apocalypse schottet 
die global agierende Umbrella Corporation eine ganze Stadt ab, um dann in einem kontrollierten Versuch die Ausbreitung des Virus und dessen Wirkung wissenschaftlich beobachten zu können. Mögliche Parallelen zu politischen Szenarien der weltweiten Corona-Pandemiebekämpfung sind zufällig: etwa, dass die britische Regierung Studierende im Herbst 2020 wieder an die Universitäten beordert hat, um der angeschlagenen Hochschul-Wirtschaft zu sicheren Mieteinnahmen zu verhelfen oder die CSU in München die WirtschaftsWiesn` erfunden hat, um der darbenden Gastronomie zu helfen und Arbeitsplätze zu sichern. Eine Pandemie kann von bestehenden Machtstrukturen missbraucht werden, genau wie es in Zombie-Fiktione dargestellt wird.

Doch auch auf persönlicher, privater Ebene zeigen Zombie-Fiktionen die Tücken einer Pandemie auf. Ist doch ein wesentlicher Bestandteil vieler Zombie-Erzählungen, das Motiv des „unerkannten Biss“. Es geht dabei um Familienmitglieder oder Freunde, die sich angesteckt haben, aber ihre Erkrankung ignorieren oder verheimlichen. In einer Variation auch gerne, dass Partner von der Erkrankung wissen, aber allen anderen Überlebenden Normalität vorspielen, um nicht die unvermeidlichen Konsequenzen ziehen zu müssen. In der Zombie-Fiktion wäre dies die unweigerliche Verwandlung der infizierten Person in einen Zombie. Das anzuratende Gegenmittel die sofortige Tötung der Person. Eine Pandemie, oder eben ein Zombieausbruch, birgt also das Problem, dass Kranke und Gesunde sich zumindest eine gefährliche Zeit lang nicht voneinander unterscheiden. Der bereits kranke Mitmensch wird zum Risiko, ohne dass man ihn oder sie erkennen könnte. Und ein willentliches Ignorieren von Krankheitssymptomen wird zum Risiko für alle in der Umgebung. Schon im Klassiker des Genres - The Night of the Living Dead [13] - findet sich dieses Motiv in der Figur der jungen Karen (Kyra Schon), die von den Zombies gebissen wurde und mit ihren Eltern im Keller eines Farmhauses ausharrt. Statt Karen zu isolieren (oder direkt zu töten), versucht die Familie sie zu pflegen und vor weiterem Schaden zu bewahren. Als sie stirbt und zum Zombie wird, wird der Keller zur Falle und Karen zum Zombie-Kind, das seine Eltern angreift und tötet.

Und letztlich zeigen Zombie-Fiktionen auch den rechtlichen Druck, den wir als Gesellschaft in einer solchen Ausnahmesituation regulieren müssen. Unser politisches System wird auf eine Probe gestellt, eine Art Sonderrecht für die Katastrophe auszurufen. Denn eine Gesellschaft, in der hochansteckende Personen sind, muss diesen Personen ihre Rechte nehmen, sie in ihrer Bewegung einschränken und sie unter Zwänge stellen - sie also etwa zwingen, Masken zu tragen oder sich nicht in größeren Gruppen zu versammeln. Dabei stellen Zombiefiktionen unsere grundlegenden Strukturen des Zusammenlebens in Frage, was die Parodie Shaun of the Dead [14] wunderbar veranschaulicht. Obwohl gerade Orte, an denen sich viele Menschen versammeln vermieden werden sollten, will der Angestellte Shaun (Simon Pegg) nicht von seinem Plan abweichen, die ZombieApokalypse möglichst ungestört in seinem Lieblingspub zu verbringen. Das Zitat im Film lautet: „Take car. Go to mum's. Kill Phil, grab Liz, go to the Winchester, have a nice cold pint, and wait for all of this to blow over. How's that for a slice of fried gold?" Natürlich ignoriert dieser Plan vollkommen das Risiko sich anzustecken und in der Tat geschieht bei der Umsetzung genau das: Shauns bester Freund Ed (Nick Frost) wird gebissen und verwandelt sich in einen Zombie. Spannend dabei ist, dass der Film einen Weg aufzeigt, wie man auf Dauer mit der Pandemie umgeht. Am Ende des Films sehen wir Shaun und Ed gemeinsam auf der Couch sitzen und weiterhin Videospiele spielen, nur das Ed jetzt ein Zombie und in Ketten gelegt ist. Selbst die Erkrankung Eds hält Shaun nicht davon ab wieder in die exakt selbe Muster zurück zu fallen, die er vor der Pandemie für normal erachtet hat. Vielleicht liegt in diesem stoischen Wunsch nach Normalität ja auch ein Hoffnungsschimmer.

Die Alternative zu einem „trotz Pandemie zurück zum alten Normal“ wäre ein „wegen der Pandemie ein neues Normal definieren“ - und hier möchte ich noch ein finales Beispiel anführen, das den Zombies den Rücken kehrt. Der Film Code 46 [15] zeigt uns eindrücklich, wie ein neues Normal aussehen könnte, das mit erhöhtem Risiko für Viren und andere Krankheiten einhergeht. Eigentlich eine Liebesgeschichte in unsicheren Zeiten, zeigt der Film eine Welt auf, in der so starke gesundheitliche Risiken existieren, dass die Bewegungsfreiheit der Menschen stark eingeschränkt werden muss. Zum einen ist der Klimawandel massiv fortgeschritten und nur wenige Gebiete der Erde sind noch unproblematisch bewohnbar. Zum anderen existieren aber auch eine Vielzahl von Umweltgiften, Infektionen und neuen, genetisch bedingten Erbkrankheiten, die eine freie Bewegung der Menschen unmöglich machen. Wer von einer sicheren Enklave zur nächsten reisen möchte, der benötigt von einer globalen Versicherungsgesellschaft ausgestellte „Papellen“, die zugleich als Visum und als Nachweis einer 
Risikobewertung gelten: Reiseversicherung und Aufenthaltsgenehmigung in einem. Angesichts von Reisewarnungen, Risikogebieten in der Pandemie und verpflichtenden Covid-Tests und Quarantäne-Maßnahmen nach Reisen erscheint diese Zukunftsvision heutzutage deutlich näher als noch vor einem Jahr. Code 46 verbindet diese neue Form der Risiko-Beurteilung mit einer globalen Wirtschaftselite, für die Mobilität zum Zeichen einer Klassenzugehörigkeit wird. Die Ungleichheit zwischen Arm und Reich verdeutlicht sich an der Leichtigkeit, mit der Impfungen und Antigene für die richtige Summe erstanden werden können. Code 46 könnte uns ein Modell dafür liefern, wie sich globale Mobilität in der Zukunft entwickelt, wie das Recht auf Freizügigkeit eingeschränkt werden wird, sollte sich ein Impfstoff doch als weniger effektiv herausstellen, als hoffnungsvoll angenommen wird.

Und damit möchte ich meine Überlegungen abschließen. Die hier vorgestellten Modelle sind weniger eindeutig, als die typischen Arbeiten im Rahmen der ASIM. Aber ich hoffe dennoch, aufgezeigt zu haben, dass bestimmte globale, komplexe System nicht das reine Modellieren von Daten für jeden sichtbar und erfahrbar werden; dass bestimmte Themen zu unberechenbar sind; und dass wir Modelle brauchen, die den Menschen näher rücken, die die Distanz zu Zahlen und Fakten überbrücken. Ich hoffe, dass die Science Fiction ihren Beitrag dazu leisten kann, dass wir Narrative finden, um wissenschaftliche Erkenntnisse verständlich zu verpacken.

\section{References}

[1] Morton, T. Hyperobjects: Philosophy and Ecology after the End of the World. Minneapolis: U of Minesota P, 2013.

[2] Schmeink, L. Repräsentation des Nicht-Erfahrbaren. Neue Rundschau. 2019 (1): 46-56.

[3] Chu, S.-Y. Do Metaphors Dream of Literal Sleep? A Science-Fictional Theory of Representation. Cambridge: Harvard UP, 2010.

[4] Gibson, W. The Peripheral. New York: Berkley, 2014.

[5] Gibson, W. Agency. New York: Berkley, 2020.

[6] Bauman, Z. Wasted Lives: Modernity and its Outcasts. Cambridge: Polity, 2004.

[7] Elysium. Regie: N. Blomkamp. Sony Pictures, 2013.

[8] Frase, P. Four Futures: Visions of the World After Capitalism. Cambridge: Verso, 2016.

[9] Centers for Disease Control and Prevention. "Zombie Preparedness” Web. 15.10.2020.

https://www.cdc.gov/cpr/zombie/index.htm.
[10] Khan, A. S. „Preparedness 101: Zombie Apocalypse.“ Centers for Disease Control and Prevention. 16.05.2011. Web. 15.10.2020. https://blogs.cdc.gov/publichealthmatters/2011/05/preparedness-101-zombie-apocalypse/

[11] 28 Weeks Later. Regie: Juan Carlos Fresnadillo. $20^{\text {th }}$ Century Fox, 2007.

[12] Resident Evil: Apocalypse. Regie: Alexander Witt. Constantin Film, 2004.

[13] The Night of the Living Dead. Regie: George A. Romero. Continental Distributing, 1968.

[14] Shaun of the Dead. Regie: Edgar Wright. Universal Pictures, 2004

[15] Code 46. Regie: Michael Winterbottom. BBC Films, 2003. 